\title{
OSCILLATIONS IN ORTHOPOSITRONIUM ANNIHILATION IN MAGNETIC FIELD IN $\mathrm{SiO}_{2}$ POWDERS
}

\author{
A.V. Berestov, A.S. Talat, V.V. Baranov, E.A. Rudak \\ AND B.A. MaRTSYNKEVICH \\ Laboratory of Nuçlear Spectroscopy, Institute of Physics \\ Academy of Sciences of Belarus \\ F. Skariny Av. 70, 220072 Minsk, Belarus
}

\begin{abstract}
The observation method of $\gamma$-quantum angular distribution oscillations in positronium annihilation in magnetic field is essentially improved. Oscillation parameters in the experiment are in agreement with theoretical estimations.
\end{abstract}

PACS numbers: 78.70.Bj, 36.10.Dr, 07.85.+n

\section{Introduction}

The phenomenon of oscillations of positronium $3 \gamma$-decay quantum angular distribution in magnetic field was predicted in papers $[1,2]$ and the idea of realization opportunity of the new matter investigating method with the use of positrons was put forward. Polarized positrons are slowed down in matter to form the polarized positronium atom. The anisotropy of the $3 \gamma$-decay quantum angular distribution relative to the positronium atom quadrupolarization will bring time oscillations of the count rate which can be experimentally observed in recording delayed coincidences between the nuclear $\gamma$-quantum $\left({ }^{22} \mathrm{Na}\right)$ and annihilation quanta.

By analogy with the $\mu$ SR-method the new technique was called the positronium spin rotation (PsSR) method [2].

The oscillation frequencies correspond to the differences in energy of Ps atom levels in the magnetic field

$$
\begin{aligned}
& \Omega_{1}=1 / 2 \Delta W\left(\sqrt{1+x^{2}}-1\right), \\
& \Omega_{2}=1 / 2 \Delta W\left(\sqrt{1+x^{2}}+1\right), \\
& \Omega_{3}=\Delta W \sqrt{1+x^{2}}
\end{aligned}
$$


where $\Delta W$ is the value of the Ps ground state hyperfine splitting, $x=4 \mu H / \Delta W=$ $0.0275 H(\mathrm{kG}), H$ is the external magnetic field strength. The oscillation can be simply detected in a weak $(x \ll 1)$ magnetic field at the frequency $\Omega_{1}$, since $\Omega_{2}, \Omega_{3} \approx 10^{12} \mathrm{~s}^{-1}$ and the terms involving $\Omega_{2}$ and $\Omega_{3}$, when averaged over the detector time resolution $\left(\approx 10^{-9} \mathrm{~s}\right)$, become zero.

The oscillation modulation depth $h_{0}$, equal to the ratio of the oscillation amplitude to value of the nonoscillating term, is defined by the following expression:

$$
h_{0}=0.213 P|\sin \Theta \sin 2 \beta \sin \alpha|,
$$

where (Fig. 1) $\beta$ and $\alpha$ are the polar and azimuthal angles specifying the direction towards the detector, $\Theta$ is the angle between the external magnetic field direction $(O Z$ axis) and the positron average polarization vector. It is assumed that the last one is lying in the plane $X Z . P$ is the degree of polarization of the positrons entering the target.
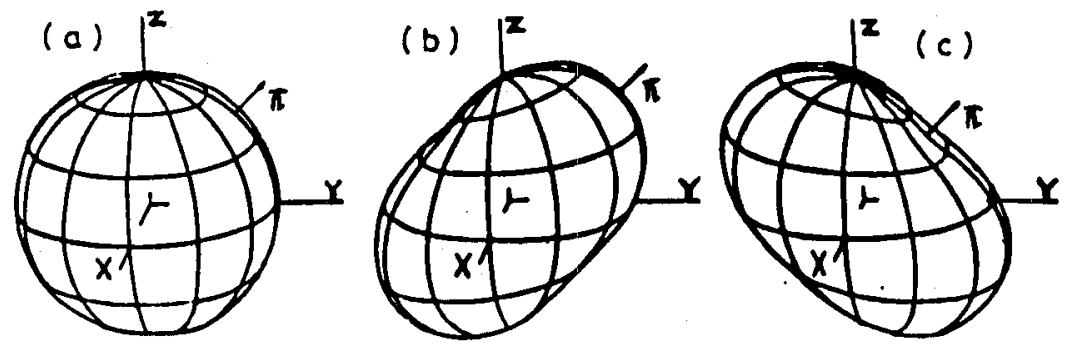

Fig. 1. The angular distribution of the orthopositronium annihilation quanta in a weak magnetic field at the time $t=0(\mathrm{a}), t=T_{0} / 4$ (b) and $t=3 T_{0} / 4$ (c); $T$ is the oscillation period, $n$ is the vector directed to the center of the detector registering the decay of $\gamma$-quanta.

The oscillation amplitude becomes maximum when the positrons entering the target are polarized perpendicular to the external magnetic field direction $(\Theta=\pi / 2)$, and the detector registering decay quanta is placed at the angle $\beta=\pi / 4$ to the magnetic field direction in the plane normal to the positron polarization vector $(\alpha=\pi / 2)$. Oscillation phases $\Psi$, observed at angles $\beta=\pi / 4$ and $\beta=3 \pi / 4$ ( $\alpha=$ const), and those observed $\mathrm{i}$ case when $\alpha=\pi / 2$ and $\alpha=-\pi / 2(\beta=$ const $)$ differ by $\pi$.

Thus, the experimentally obtained time distribution is described in first approximation by the expression

$$
N(t)=A \exp (-t / \tau)\left[1+h_{0} \sin \left(2 \pi t / T_{0}+\Psi\right)\right]+F,
$$

where $A$ is the amplitude of the nonoscillating term, $\tau$ is the orthopositronium lifetime, $T_{0}=2 \pi / \Omega_{1}$ is the oscillation period, $F$ is background.

The results of the first experiments for observation of $\gamma$-quanta angular distribution in orthopositronium annihilation in magnetic field are given in [3] and confirmed in [4]. But the observation time in the above-mentioned experiment [3] was about 50 hours. This article describes advanced observation technique and the results of the new experiment. 


\section{Experimental results}

To register the time spectra of orthopositronium annihilation, we made use of the system based on three $\mathrm{NaI}(\mathrm{Tl})$ detectors $(\oslash 150 \times 100 \mathrm{~mm})$ [5] included into the delayed coincidence circuit. One of the detectors is set to register a nuclear $\gamma$-quantum of $1275 \mathrm{keV}\left({ }^{22} \mathrm{Na}\right)$, and other two, facing each other and being incorporated into the summation circuit, detect annihilation quanta. The time resolution of the system is $7 n \mathrm{~ns}$, time range is $500 \mathrm{~ns}$.

The "positronium producing" target used was a tablict of fine-grained $\mathrm{SiO}_{2}$, a pressure was $(2 \pm 0.2) \times 10^{-2} \mathrm{~Pa}$. The magnetic field was generated by the $\mathrm{SmCo}$ $40 \times 40 \times 4 \mathrm{~mm}$ magnets, its homogeneity over the target volume was $\Delta H / H \approx 5 \%$.

The intensity of positron source $\left(2 \mathrm{~mm}\right.$ in diameter) is $3 \times 10^{5} \mathrm{~Bq}$. The positrons are polarized parallel to the direction of their motion to the degree $P=v / c$, where $v$ is the velocity of a positron at the time of its escape from the source. The value of the positron polarization averaged over the source energy spectrum is $P=0.65$. Owing the fact that the positrons enter the target (and hence, become polarized) within the solid angle $2 \pi$, the average degree of positron polarization $P$ decreases to one half.

To decrease the background to the positron annihilation ratio, the Ps time spectra were registered during the time interval $t=25 \ldots 500 \mathrm{~ns}$.

We had used the fact that oscillation phases $\Psi$ observed in constant magnetic field $H$ at angles $\beta_{1}=\pi / 4$ and $\beta_{2}=3 \pi / 4(\alpha=\pi / 2)$ differ by $\pi$. If we subtract such time distributions we receive the distribution defined by the following expression (see Eq. (3)):

$$
N(t)=2 A \exp \left(-t / \tau_{0}\right) h_{0} \sin \left(2 \pi t / T_{0}+\Psi\right) .
$$

The experimentally obtained data was analyzed with the non-linear least squares method using the hypothesis that the time distribution is described by Eq. (4). Magnitude of $I$, theoretical values $\Psi_{T}, T_{T}$ and values of $T_{0}, A, h_{0}, \tau, \Psi$, estimated with the least squares method, are given in Table. In this Table there are also given the Pirsons accordance parameters $\chi^{2}$ and their averaged values $\bar{\chi}^{2}$ equal to the number of experimental points minus the number of selected parameters. The experimental time distributions are given in Fig. 2.

TABLE

The results of analyzing the experimental data with the non-linear least squares method.

\begin{tabular}{c|c|c|c|c|c|c|c|c|c|c}
\hline \hline$N$ & $H[\mathrm{kG}]$ & $\Psi_{T}$ & $T_{T}[\mathrm{~ns}]$ & $T_{0}[\mathrm{~ns}]$ & $A \times 10^{-3}$ & $h_{0} \times 10^{2}$ & $\tau[\mathrm{ns}]$ & $\Psi$ & $\chi^{2}$ & $\bar{\chi}^{2}$ \\
\hline 1 & 0.68 & $\pi$ & $52 \ldots 61$ & $58.6 \pm 0.2$ & $480 \pm 1$ & $\mathbf{3 . 0} \pm 0.1$ & $100 \pm \mathbf{3}$ & $\pi$ & 99 & 95 \\
2 & 0.61 & $\pi$ & $61 \ldots 73$ & $68.6 \pm 0.4$ & $330 \pm 1$ & $2.7 \pm 0.3$ & $108 \pm 10$ & $\pi$ & 95 & 95 \\
3 & 0.58 & 0 & $73 \ldots 86$ & $81.4 \pm 0.4$ & $270 \pm 1$ & $2.6 \pm 0.2$ & $98 \pm 7$ & 0 & $\mathbf{5 5}$ & 95 \\
4 & 0.48 & 0 & $102 \ldots 123$ & $109.2 \pm 1.2$ & $360 \pm 1$ & $2.9 \pm 0.3$ & $117 \pm 13$ & 0 & $\mathbf{2 5 8}$ & 91
\end{tabular}

As can be seen from Table, the period and the observed oscillation phase are in good agreement with the magnitude and direction of the magnetic field induction. The theoretically estimated value $h_{0} \approx 0.035$ is little higher than the experimental results. This fact takes place probably due to the neglected effects of predominant absorption of soft $\gamma$-quanta by the magnet material, magnetic field inhomogeneity, and the relaxation of positronium polarization in the target. 


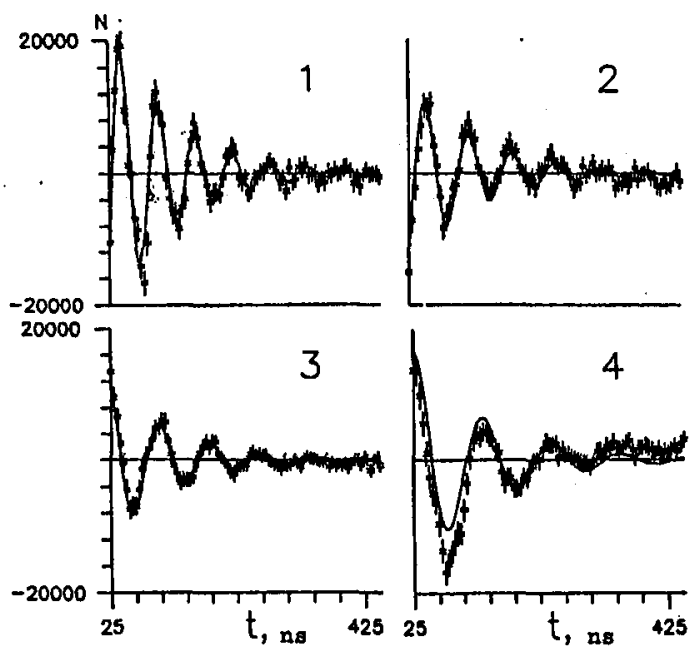

Fig. 2. The time distributions obtained by subtraction of the distributions observed at angles $\beta_{1}=\pi / 4$ and $\beta_{2}=3 \pi / 4(\alpha=\pi / 2)$. The solid curve shows the theoretically estimated time distribution.

\section{Conclusions}

Thus, using more intensive $\left({ }^{22} \mathrm{Na}\right)$ source, magnet system with a low absorption of decayed $\gamma$-quanta comparatively [3] and the method of subtraction of the distributions with oscillation phases differing by $\pi$ we essentially improved the observation conditions of $\gamma$-quantum angular distribution oscillations in positronium annihilation in magnetic field. The exposition time of experimental spectra necessary for obtaining the oscillation parameters is several hours.

The work was fulfilled under support of Foundation of Fundamental Research of the Republic of Belarus.

\section{References}

[1] V.G. Baryshevsky, Dokl. Akad. Nauk BSSR 16, 445 (1966).

[2] V.G. Baryshevsky, Phys. Status Solidi B 124, 619 (1984).

[3] V.G. Baryshevsky, O.N. Metelitsa, V.V. Tikhomirov, S.K. Andrukhovich, A.V. Berestov, B.A. Martsinkevich, E.A. Rudak, Phys. Lett. A 136, 428 (1989).

[4] S. Fan, C.D. Beling, S. Fung, in: Book of Abstracts of 10th Int. Conf. on Positron Annihilation, Beijing (China) 1994, p. 216.

[5] A.V. Berestov, F.E. Zyazyulya, Prib. Tekn. Eksp. 5, 47 (1983). 